\title{
Commentary: Tag, you're it! Finding and treating early lung cancers in a single setting
}

\author{
Ngoc-Quynh Chu, MD, and Yolonda L. Colson, MD, PhD
}

\author{
From the Division of Thoracic Surgery, Department of Surgery, Brigham and Women's Hospital, Boston, Mass. \\ Disclosures: Stryker (Novadaq Industries) has provided an equipment loan for independent studies by \\ Y.L.C. Y.L.C. has a sponsored research agreement with Canon USA for an unrelated project. Remaining author \\ has nothing to disclose with regard to commercial support. \\ Received for publication Dec 19, 2018; accepted for publication Dec 19, 2018; available ahead of print Feb 7 , \\ 2019. \\ Address for reprints: Yolonda L. Colson, MD, PhD, Division of Thoracic Surgery, Brigham and Women's Hos- \\ pital, 75 Francis St, Boston, MA 02115 (E-mail: ycolson@ partners.org). \\ J Thorac Cardiovasc Surg 2019;157:e217-8 \\ $0022-5223 / \$ 36.00$ \\ Copyright (c) 2019 by The American Association for Thoracic Surgery \\ https://doi.org/10.1016/j.jtcvs.2018.12.069
}

The increasing use of computed tomographic (CT) imaging and the advent of lung cancer screening has allowed the detection of potential cancers in the very early stages of disease. The size and characteristics of these radiologically visible pulmonary nodules (particularly ground-glass opacities), however, mean that they are often neither visible nor palpable in the operating room, making accurate tissue sampling and diagnosis uncertain and resection difficult. This challenge makes preoperative lesion localization crucial and involves image guidance with CT or electromagnetic navigation (EN) bronchoscopy, in conjunction with labeling by physical markers (hook wires, microcoils, fiducials) or dyes (patent blue, indocyanine green). Additional intraoperative methods to confirm accurate localization are often needed as well (fluoroscopy, ultrasound, near-infrared imaging). ${ }^{1}$

In this issue of the Journal, Hsu and colleagues ${ }^{2}$ describe a method for lesion localization that uses an EN-guidance system for percutaneous microcoil placement to mark a nodule for immediate thoracoscopic biopsy. Unlike preceding EN systems, the described technology allows both bronchoscopic and transthoracic guided routes of lung access. Hsu and colleagues ${ }^{2}$ leverage the transthoracic capability to navigate to the lesion and deploy a microcoil through the biopsy needle to mark the target lesion. In most of the cases presented, a dye was also administered with the microcoil for dual localization. Immediate video-assisted thoracoscopic surgical biopsy was done, with additional intraoperative imaging to confirm marker location before resection. In all cases, both the microcoil and the lesion of interest were within the resected specimen, with a mean microcoil-to-lesion distance of $4.2 \mathrm{~mm}$.

Numerous groups have reported EN bronchoscopy for pleural dye marking of lesions for surgical resection, tissue collection for molecular profiling, fiducial marker placement to guide stereotactic body radiation therapy, and indocyanine green and near-infrared image-guided mediastinal lymph node sampling. ${ }^{3}$ The application of EN

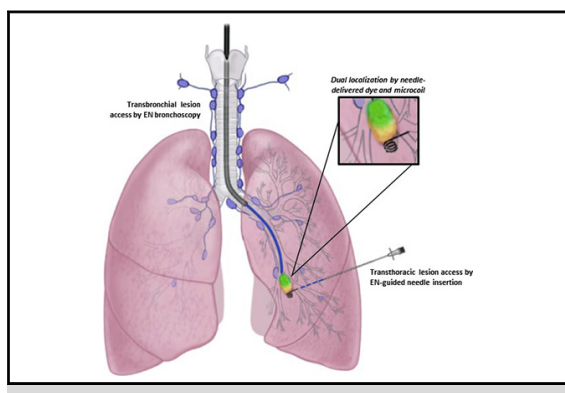

Routes and markers for localizing small lung cancers by electromagnetic navigation (EM)

\section{Central Message}

Lung cancer diagnosis has become challenging as smaller nodules and ground-glass opacities are difficult to identify or palpate. New intraoperative technologies can be used to locate these lesions.

See Article page e211.

guidance for microcoil placement as reported by Hsu and colleagues $^{2}$ promises multiple benefits and is an innovative use of existing technology. Currently, microcoils are placed percutaneously under CT guidance to mark the tumor site before the patient is transported to the operating room for resection. The novelty in this report lies in percutaneous microcoil placement by EN guidance in the operative suite, allowing immediate video-assisted thoracoscopic surgical resection. A major advantage of this approach is the streamlined workflow and condensed procedure time, with microcoil placement and video-assisted thoracoscopic surgical biopsy performed during the same procedure, in the same room, and by the same team. This minimizes radiation exposure, likelihood of microcoil displacement (a not infrequent complication), and potentially hospital costs while rendering irrelevant the usual concern for pneumothorax after transthoracic biopsy or coil placement in the radiology suite. The addition of transthoracic capabilities to an EN bronchoscopy system is a useful feature, providing an additional means for biopsy, particularly when the diagnostic yield of transbronchial EN bronchoscopy remains generally lower than that of transthoracic needle biopsy. ${ }^{3}$ Although the number of patients reported in this study is small, safety and feasibility of what will become an increasingly common approach to identify and resect small nodules in the lung is demonstrated, and this will be a valuable technique in the 
treatment of early-stage lung cancers at a size not previously possible.

\section{References}

1. Lin MW, Chen JS. Image-guided techniques for localizing pulmonary nodules in thoracoscopic surgery. J Thorac Dis. 2016;8(Suppl 9):S749-55.
2. Hsu PK, Wu YC. The feasibility of electromagnetic navigationguided percutaneous microcoil localization for thoracoscopic resection of small pulmonary nodules. J Thorac Cardiovasc Surg. 2019;157: e211-4.

3. Mehta AC, Hood KL, Schwarz Y, Solomon SB. The evolutional history of electromagnetic navigation bronchoscopy: state of the art. Chest. 2018;154: 935-47. 